\title{
Multiple Morphologies of Gold-Coated Magnetite Nanoparticles Are Conjugated With Ligands and Can Produce Receptor-Mediated Biological Effects.
}

\author{
Evan S. Krystofiak ${ }^{1}$, Eric C. Mattson ${ }^{2}$, Ralph M. Albrecht ${ }^{3,4,5}$, Marija Gajdardziska-Josifovska ${ }^{2}$ and Julie
} A. Oliver ${ }^{1,3}$.

1. Department of Biological Sciences, University of Wisconsin-Milwaukee, Milwaukee, WI 53211 USA.

2. Department of Physics and Laboratory for Surface Studies, University of Wisconsin-Milwaukee, Milwaukee, WI 53211 USA.

3. Departments of Animal Sciences, ${ }^{4 .}$ Pharmaceutical Sciences and ${ }^{5 .}$ Pediatrics, University of Wisconsin, Madison, WI 53706 USA.

Gold-coated magnetite nanoparticles have significant utility in biological applications where particles possessing both magnetic susceptibility and stability at physiological $\mathrm{pH}$ and salt concentrations are required. However, reported synthesis procedures give inconsistent results among investigators and even between batches prepared by a single investigator. A great deal of effort has been devoted to creating nanoparticles of uniform size and shape that consist of a magnetite core completely surrounded by a thin gold shell. This morphology has been described as a "core-shell," and has remained elusive. Our previous work suggests that a true core-shell morphology is not absolutely required for gold-coated magnetite nanoparticles to be functionalized with biologically relevant molecules [1]. A minority of the population of the stock nanoparticle sols are aggregates composed of agglomerated magnetite nanoparticles that are incompletely coated with gold. Following functionalization with ligands or antibodies and centrifugation to separate conjugated nanoparticles from free protein, a protein layer is observed around both the gold coating and the exposed magnetite found in aggregates. The purpose of this study was to determine whether the aggregated nanoparticles behave equivalently to isolated nanoparticles in biological systems, or if avoiding the preparation and/or inclusion of this minority population is important to the successful application of gold-coated magnetite nanoparticles in diagnostics and therapeutics as contrast, labeling, or targeting tools.

To compare the biological function of isolated and aggregated nanoparticles, conjugates were prepared with human fibrinogen [1]. The binding of fibrinogen conjugates to its integrin $\alpha \operatorname{IIb} \beta 3$ receptor on surface-activated human platelets and the actin cytoskeleton-dependent translocation of fibrinogencross-linked receptors in the platelet membrane [2] were used to indicate biological activity. The percentages of aggregated nanoparticles found in the stock sol, in the fibrinogen-conjugated suspension, and bound to the platelet surface were compared. Drops of the stock and fibrinogen-conjugated goldcoated magnetite nanoparticles were spotted onto EM grids and examined in a Hitachi H-600 TEM at 75 $\mathrm{kV}$. Isolated human platelets were activated by adherence to EM grids, labeled with fibrinogenconjugated nanoparticles, and prepared for electron microscopy. Samples were not stained or sputtercoated with heavy metals to avoid interference with nanoparticle identification. Platelet specimens were examined in either a Hitachi H-600 TEM at $75 \mathrm{kV}$ or a Hitachi S-4800 SEM at $5 \mathrm{kV}$ using a photodiode backscattered electron detector. The percentage of aggregated particles was scored in 200-1500 nanoparticles in 2-5 randomly selected fields per sample. Nanoparticle conjugates that had translocated to the most central regions of the platelet membranes were too closely spaced for accurate scoring, and were excluded. Nanoparticles otherwise obscured, for example by membrane folds, were also excluded. Statistical analysis was by chi-square. 
The percentages of aggregated nanoparticles in samples prepared over the course of a typical experiment are shown in Table 1. The proportion of aggregates decreased with successive steps $(\mathrm{p}<<0.001)$. The stock sol contained twice as many aggregates as the protein conjugates. Loss of aggregates most likely occurred during centrifugation to separate free protein from nanoparticle conjugates, as evidenced by a hard pellet that did not resuspend. Half as many aggregates were found on the platelet surface as in the fibrinogen conjugates, potentially indicating that they interact with receptors less effectively than isolated nanoparticles. However, it is possible that aggregates bound receptors equally well as isolated nanoparticles, but were preferentially recruited to internal membranes and/or the most central areas of the platelets, and were thus excluded from analysis. Figure 1 shows that aggregated nanoparticles bound to the fibrinogen receptor triggered translocation in the membrane similarly to the isolated nanoparticles, suggesting that they are functionally equivalent. SEM and TEM analysis gave equivalent results. These data demonstrate that multiple morphologies of gold-coated magnetite can be functionalized for activity and be used to induce or monitor specific receptor-mediated biological effects.

References:

[1] ES Krystofiak et al., Microsc. Microanal. 18 (Suppl 2) (2012) 1650.

[2] RM Albrecht et al., in "The Science of Biological Specimen Preparation," ed. M Mueller et al., (SEM Inc., AMF O’Hare) (1985) p. 185.

[3] This work was supported by a Scientist Development Grant (9030253N) from the American Heart Association (JAO), and an MRI grant (0723002) from the National Science Foundation (MGJ).

\begin{tabular}{cccc}
\hline Sample & Isolated & Aggregated & \% Aggregated \\
\hline Stock Nanoparticles & 256 & 37 & 12.6 \\
$\begin{array}{c}\text { Fibrinogen-conjugated } \\
\text { Nanoparticles }\end{array}$ & 853 & 61 & 6.7 \\
$\begin{array}{c}\text { Platelet-bound Nanoparticles, } \\
\text { SEM Analysis }\end{array}$ & 1449 & 47 & 3.1 \\
$\begin{array}{c}\text { Platelet-bound Nanoparticles, } \\
\text { TEM Analysis }\end{array}$ & 579 & 21 & 3.5 \\
\hline
\end{tabular}

Table 1. Isolated and aggregated gold-coated magnetite nanoparticles present before and after conjugation with fibrinogen, and after conjugated nanoparticles were used in labeling.
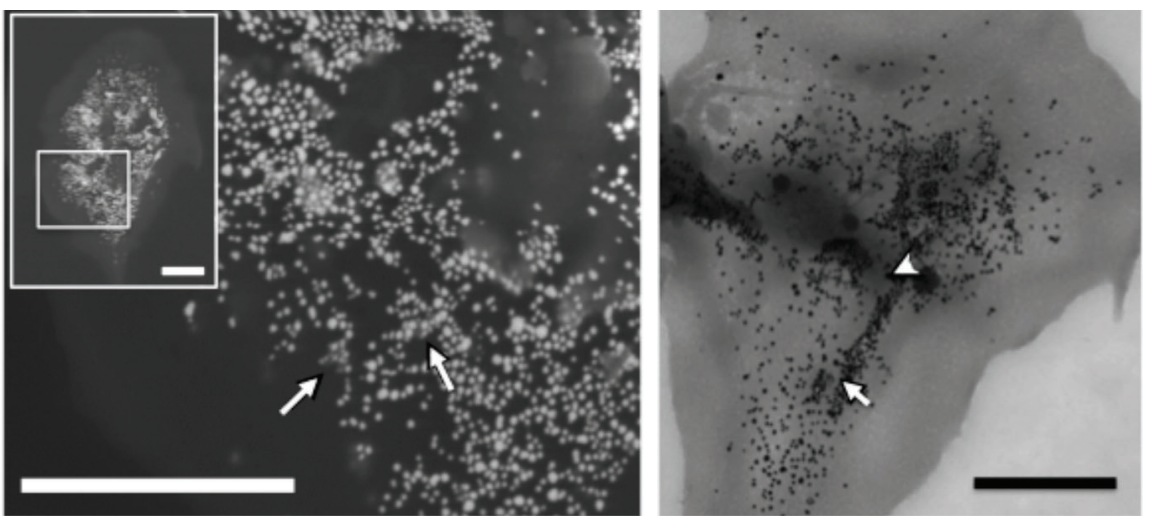

Figure 1. Arrows show examples of aggregated nanoparticles used in cell labeling. Left, backscattered electron SEM image of platelet labeled with fibrinogen-conjugated, gold-coated magnetite. Inset shows the entire platelet; box indicates area shown at higher magnification. Right, TEM image of labeled platelet. Arrow, example of aggregated nanoparticles; arrowhead, area containing nanoparticles in the platelet internal membrane system which were excluded from analysis. Size bars $=1 \mu \mathrm{m}$. 